\title{
Sexual acting out: Diagnostic category or moral judgment?
}

\author{
RUSSELL EISENMAN \\ Temple University, Philadelphia, Pennsylvania
}

\begin{abstract}
Is the term sexual acting out used only to describe something about the patient, or does it have an implicit moral condemnation as well? To clarify the implicit meaning of the term, its use was studied at a state mental hospital and at a university psychology clinic. Significant findings included the following: (1) sexual acting out was seldom used to describe males, and was almost exclusively applied to females, and (2) sexual acting out was used in a negative, condemning way. Implications of the results are discussed.
\end{abstract}

All societies have norms that are enforced with penalties against those who deviate. In fact, the deviant is subject to such penalties as scorn, scapegoating, imprisonment, loss of status, physical attack, being called "crazy," or murder. What is normal in one society may be considered insane behavior in another society, so the deviant behavior is not necessarily something the person possesses, but is the result of his/her behavior being sufficiently different to be labeled.

The present study is concerned with the way hospitals and clinics respond to people said to have engaged in sexual acting out. Criticisms have been made against the mental hospital or the university clinic on the grounds that such institutions serve as social control agencies for society, suppressing those who engage in what is defined as deviant behavior. Although there have been several case studies and theoretical reports on this phenomenon, little data has been gathered to support this criticism. The present investigation was undertaken to see if a state mental hospital and a university psychology clinic would, in fact, reflect the prejudices of the culture by the way in which the personnel at these institutions respond to those accused of sexual acting out. It seemed necessary to focus on one area, sexual acting out, rather than to try to understand all the ways in which psychiatric or psychological institutions reflect the biases of the culture. If it could be shown that these agencies' responses to sexual acting out are consistent with cultural biases, then further data gathering to determine whether these agencies act as social control forces would seem reasonable. If, on the other hand, the evidence did not uphold the criticism, some doubt would be cast on the critique.

In 1914, Freud (1946) wrote that acting out may occur in psychoanalysis when the patient expresses in action what is forgotten or repressed. Various amplifications and correlates of acting out have been considered (Altman, 1957; Fenichel, 1945; Greenacre, 1950; Kanzer, 1957; Rosenfeld, 1966), but the concept seems to be used in a

The author's address is Department of Psychology (265-67), Temple University, Philadelphia, PA 19122. vague, ill-defined way on many occasions. Of special interest is the use of the concept sexual acting out. When applied to sexual behavior, the concept is even more amorphous than is the general concept of acting out, perhaps because sexual behavior is a highly charged area about which many people have conflicting or distorted views. One way to understand the meaning of a term is to see how people use it. Despite what people say, if a term is always used with a certain connotation, then that connotation may be said to be part of the implicit meaning. By noting the way in which sexual acting out was employed in two clinical settings, the present study sought to elucidate the implicit meaning attached to the term.

\section{METHOD}

An assistant and I recorded all instances of the use of the term sexual acting out at (a) a state mental hospital staffed by psychologists, psychiatrists, and social workers, and (b) a university psychology clinic attended by psychology graduate students and one or more professors. A different assistant was used for each institution. The assistants were instructed to record the following on a note pad each time the phrase sexual acting out or its equivalent (e.g., acting out in a sexual manner) was used: status of person making statement (graduate student, professor, social worker, psychiatrist, etc.), sex of speaker and of patient referred to, final diagnosis of patient referred to, and general tone of statement (favorable, unfavorable, neutral). Any additional information that seemed useful was to be included (i.e., if patient was described as suicidal, homosexual, etc.). The assistants and I had over $90 \%$ agreement on recording the use of sexual acting out or its equivalent, and $100 \%$ agreement on accurately recording the sex of speaker and of patient. Over $90 \%$ agreement between the two assistants and me occurred for general tone of the statement and for final diagnosis. For both the state mental hospital and the university psychology clinic, the statements were noted over a period of 3 months.

\section{RESULTS}

At the state mental hospital, 40 statements were recorded over the 3-month period as referring to sexual acting out or its equivalent, whereas at the university psychology clinic, the concept was used 32 times during the 3-month study. The concept was not used more frequently by one kind of professional than another, or by one sex 
of speaker over another. Also, final diagnosis, in the sense of the psychiatric classification system (American Psychiatric Association, 1980), was unrelated to use of the term: there was no greater likelihood that psychotics, neurotics, sociopaths, or other classifications were described as having acted out sexually. However, two findings did show statistical significance. Sexual acting out was usually reported for female patients: at the state mental hospital, the term was used for 25 different patients, 20 females and 5 males ( $p=.004$, two-tailed binomial test). At the university clinic, the term was used for 20 patients, 16 females and 4 males ( $p=.012$, two-tailed binomial test).

The general tone of the speaker who referred to sexual acting out was seldom positive. Thirty-five of the 40 hospital statements were rated as negative, in that the statement implied criticism or condemnation, whereas the other 5 were rated as neutral. This preponderance of negative over neutral statements yields $\chi^{2}(1)=22.50$, which is significant well beyond the .001 level, and actually underestimates the relationship, since none of the statements were positive. For the 32 statements at the university clinic, 2 were rated positive, 3 neutral, and the remaining 27 negative. Comparing the negative statements with neutral and positive statements combined yielded $\chi^{2}(1)=15.12$, which is significant beyond the .001 level.

Of the 9 males described as having sexually acted out, 5 were said to be homosexuals. Although such a finding is suggestive rather than conclusive, due to the small number of subjects involved, it is consistent with the findings presented above that sexual acting out is most often applied to females. In popular thinking, homosexuality in males suggests an absence of masculinity. The findings suggest that the phrase sexual acting out is seldom used to describe male patients, and, when it is, the probability is high that the patient will also be considered homosexual.

\section{DISCUSSION}

The fact that the term sexual acting out has a negative connotation could have been predicted from the clinical writings cited in the introduction. However, the sex bias, which is evident because the concept is applied almost exclusively to females, is a less obvious finding and has implications for the implicit meaning of the term. Female sexual behavior is subject to more condemnation in our society than is male sexual behavior, as is implied by the sexual double standard that implies that it is all right for males to do things that are not acceptable behaviors for females. Furthermore, it appears that, in fact, female sexual behavior is more inhibited (Kinsey, Pomeroy, \& Martin, 1948; Kinsey, Pomeroy, Martin, \& Gebhard, 1953). One way in which society maintains its current standards is by condemnation of deviants (Eisenman, 1967).
As Szasz (1960) has pointed out, many of the terms that we use in the diagnostic process should be looked upon as referring to interpersonal behavior rather than to disease. This interpersonal behavior is governed by the mores present in the society, and it would appear from this perspective that sexual acting out is a moral judgment as well as a diagnostic indicator. The two are not incompatible. As a diagnostic indicator, sexual acting out refers to certain aspects of the patient's behavior. But, as a moral judgment, sexual acting out is a term that allows the clinician to condemn the patient while seeming to be impersonal or objective.

A final methodological warning is in order. Although the findings were similar for the hospital and the university clinic studied, variation in the implicit meaning of sexual acting out should occur as other hospitals, clinics, or different institutions are studied. The number of statements on the concept were relatively few, even though the institutions were studied for a 3-month period. Since sexual acting out, or its equivalent, was used to refer to a single patient more than once (e.g., one psychologist used the term four times to refer to a patient), the binomial tests for sex of patient referred to were based on the number of patients rather than on the number of statements. This counting of patients further reduced the number of statements, but the results were consistent with the belief that sexual acting out is a term that not only diagnoses the patient, in the sense of telling us something about him/her, but also morally condemns the person. Clinicians seem to share the stereotyped views of society regarding sexual behavior by females, at least to the extent that clinicians have an implicit double standard about sexual acting out. That there is some evidence of use of the concept as a moral condemnation against homosexual males as well as against females is provocative and worthy of more investigation to determine if and how clinicians morally condemn homosexuals.

\section{REFERENCES}

Altman, L. (1957). On the oral nature of acting out. Journal of the American Psychoanalytic Association, 5, 648-662.

American Psychiatric Association. (1980). Diagnostic and statistical manual of mental disorders (3rd ed.). Washington, DC: Author.

EISENMAN, R. (1967). Scapegoating the deviant in two cultures. International Journal of Psychology, 2, 133-138.

Fenichel, O. (1945). Neurotic acting out. Psychoanalytic Review, 32, 197-206.

FREUD, S. (1946). Recollection, repetition, and working through. In Collected papers. London: Hogarth Press. (Original work published 1914)

GreenaCre, P. (1950). General problems of acting out. Psychoanalytic Quarterly, 19, 445-467.

KANZER, M. (1957). Acting out and its relation to the impulse disorders. Journal of the American Psychoanalytic Association, 5, 136-145.

Kinsey, A. C., Pomeroy, W. B., \& Martin, C. E. (1948). Sexual behavior in the human male. Philadelphia: Saunders.

Kinsey, A. C., Pomeroy, W. B., Martin, C. E., \& Gebhard, P. H. (1953). Sexual behavior in the human female. Philadelphia: Saunders.

Rosenfeld, H. A. (1966). The need of patients to act out during analysis. Psychoanalytic Forum, 1, 20-25.

Szasz, T. (1960). The myth of mental illness. American Psychologist, 15, 113-118.

(Manuscript received for publication March 3, 1987.) 